\title{
Schisandrin B protects against myocardial ischemia/reperfusion injury via the PI3K/Akt pathway in rats
}

\author{
XUYONG ZHAO $^{1 *}$, YIJIA XIANG ${ }^{1 *}$, CHANGHONG CAI $^{1}$, AIMING ZHOU $^{1}$, NING ZHU $^{1,2}$ and CHUNLAI ZENG $^{1}$ \\ ${ }^{1}$ Department of Cardiology, The Fifth Affiliated Hospital of Wenzhou Medical University, Lishui Central Hospital, \\ Lishui, Zhejiang 323000; ${ }^{2}$ Department of Cardiology, The Third Clinical College of Wenzhou Medical University, \\ Wenzhou People's Hospital, Wenzhou, Zhejiang 325000, P.R. China
}

Received March 23, 2017; Accepted September 14, 2017

DOI: $10.3892 / \mathrm{mmr} .2017 .7926$

\begin{abstract}
The natural medicinal monomer, schisandrin B (Sch B), has been shown to exert cardioprotective effects; however, the underlying mechanisms of these effects remain to be fully elucidated. Therefore, the aim of the present study was to investigate whether Sch B attenuated myocardial ischemia/reperfusion (I/R) injury via the phosphoinositide 3-kinases (PI3K)/Akt signaling pathway. To confirm this, I/R models were established in rats by ligation of the left anterior descending coronary artery. A group of animals were administered with Sch B (60 mg/kg, lavage) and/or the PI3K inhibitor, LY294002 (0.3 mg/kg, intraperitoneal). Myocardial infarct size, myocardial infarct serum markers, myocardial apoptotic index and the expression of Akt were measured in each group. The results demonstrated that the administration of Sch B reduced the size of the myocardial infarct, and this effect was eliminated following LY294002 treatment. In addition, the administration of Sch B decreased the apoptotic index and the serum markers of myocardial infarction. Sch B administration also increased the expression of phosphorylated Akt, and Sch B treatment decreased the B-cell lymphoma 2 (Bcl-2)-like protein 4/Bcl-2 ratio and the expression of cleaved caspase-3. Therefore, Sch B may protect myocardial tissue from I/R injury via the PI3K/Akt signaling pathway in rats.
\end{abstract}

\section{Introduction}

Acute myocardial infarction (AMI) is one of the most life threatening diseases in the modern world in terms of its high

Correspondence to: Professor Chunlai Zeng, Department of Cardiology, The Fifth Affiliated Hospital of Wenzhou Medical University, Lishui Central Hospital, 289 Kuocang Road, Lishui, Zhejiang 323000, P.R. China

E-mail: zengchunlai@medmail.com.cn

${ }^{*}$ Contributed equally

Key words: schisandrin B, ischemia/reperfusion injury, phosphoinositide 3-kinase/Akt pathway, LY294002, apoptosis mortality rate. The most successful therapeutic strategy, in which coronary circulation is restored, was established to provide essential preservation of viable myocardium following AMI (1). However, reperfusion therapy may induce oxidative stress and inflammatory responses, which causes cell damage and can lead to death. This pathophysiological process has been defined as ischemia/reperfusion (I/R) injury (2). To alleviate this injury, numerous investigations have focused on interventions, which can attenuate I/R injury (3); however, the conversion of these strategies into clinical practice has not been satisfactory. To improve the clinical efficacy of AMI, it is important to identify novel pharmaceutical preparations for the prevention of myocardial I/R injury.

As a form of traditional medicine, Schisandra chinensis has been used extensively in East Asia for thousands of years and several symptoms can be alleviated with its use $(4,5)$. Schisandrin B (Sch B) is the natural medicinal monomer extracted from Schisandra chinensis, and has been confirmed in several studies to have multiple functions, including an antioxidant effect on the liver $(6,7)$. In addition, studies have found that Sch B protects cerebral tissue from ischemia and I/R injury $(8,9)$. It has also been reported that Sch B can attenuate myocardial I/R injury in vitro (10); however, the precise mechanism underlying the effects of Sch B in vivo remain to be elucidated.

The phosphoinositide 3-kinases (PI3K)/Akt (also known as $\mathrm{PKB}$ ) signaling pathway is considered to be involved in the regulation of cellular activation and apoptosis (11). Investigations have indicated that the PI3K/Akt signaling pathway can elevate cellular viability by limiting the apoptotic events induced by noxious stimuli (12). When the PI3K/Akt signaling pathway is activated, the myocardial tissue injury induced by $\mathrm{I} / \mathrm{R}$ is relieved (13).

In the present study, an I/R rat model was established to investigate whether Sch B protected myocardial tissue from I/R injury by suppressing apoptosis, and to determine the possible mechanisms underlying the cardioprotective effects of Sch B in vivo.

\section{Materials and methods}

Animalmodels andexperimentaldesign.Male Sprague-Dawley (SD) rats (180-200 g, 7 weeks old) were purchased from the 
Experimental Animal Center of Zhejiang Province (Zhejiang, China). The involvement of animals in the experiments was reviewed and approved by the Animal Policy and Welfare Committee of Wenzhou Medical University (Wenzhou, China). All animals were housed in groups of 4 rats per cage and maintained in controlled conditions ( $12 \mathrm{~h}$ light-dark cycle; $20-24^{\circ} \mathrm{C} ; 45-55 \%$ humidity). Food and water were available ad libitum.

Chemicals and reagents. Sch B was obtained from Xi'an Plant Bio-Engineering Co., Ltd. (Xi'an, China). LY294002, a PI3K inhibitor, was obtained from Sigma-Aldrich; Merck Millipore (Darmstadt, Germany). Anti-phosphorylated (p)-Akt (Ser473; cat. no. 4060), anti-Akt (cat. no. 4685), anti-B-cell lymphoma 2 (Bcl-2; cat. no. 2876), anti-cleaved caspase-3 (cat. no. 9661), anti-GAPDH (cat. no. 5174) antibodies, and anti-rabbit horseradish-peroxidase (HRP)-conjugated immunoglobulin G (cat. no. 7074) secondary antibodies were purchased from Cell Signaling Technology, Inc. (Danvers, MA, USA). Anti-Bcl-2-like protein 4 (Bax; cat. no. sc-6236) antibody was purchased from Santa Cruz Biotechnology, Inc. (Dallas, TX, USA).

Experimental groups. According to previous studies $(9,10)$, preliminary experiments to assess three doses of Sch B $(10,30$ and $60 \mathrm{mg} / \mathrm{kg}$ ) showed that $60 \mathrm{mg} / \mathrm{kg} \mathrm{Sch} \mathrm{B} \mathrm{was} \mathrm{the}$ optimal dose based on the myocardial infarction size $(81.3 \pm 7.1$, $74.3 \pm 6.7$ and $62.8 \pm 11.2 \%$, respectively). In the subsequent experiments, the rats were randomly divided into five groups: i) Sham group; ii) I/R group; iii) $\mathrm{Sch} \mathrm{B}+\mathrm{I} / \mathrm{R}$ group; iv) $\mathrm{Sch}$ $\mathrm{B}+\mathrm{LY}+\mathrm{I} / \mathrm{R}$ group; and v) $\mathrm{LY}+\mathrm{I} / \mathrm{R}$ group. The $\mathrm{Sch} \mathrm{B}$-treated rats were administered with Sch B $(60 \mathrm{mg} / \mathrm{kg}$ intragastrically, dissolved/suspended in olive oil, once a day) for 15 days, and the remaining rats received olive oil only. The LY-treated rats were administered with LY294002 [0.3 mg/kg dissolved in dimethyl sulfoxide (DMSO; 0.1\%)] 30 min prior to reperfusion.

Establishment of myocardial I/R model. Following sacrifice of the rats with intraperitoneal pentobarbital $(60 \mathrm{mg} / \mathrm{kg})$, the rats were mechanically ventilated using a rodent respirator (DW-3000B; Huaibei Zhenghua Technology Co., Ltd., Anhui, China). A limb lead II electrocardiogram (ECG) was continuously monitored and recorded during the entire procedure. Following cardiac exposure, the left anterior descending (LAD) coronary artery was ligated with a 6-0 polypropylene suture below the inferior margin of the left auricle. At the top of the vessel, a vinyl tube was placed for reversible coronary occlusion. The success of ischemia was judged by the ST-segment elevation on the ECG. The myocardium underwent ischemia for $45 \mathrm{~min}$, following which the slipknot was released for $24 \mathrm{~h}$ to mimic reperfusion.

Assessment of myocardial infarction size. Infarction size was evaluated using Evans Blue (EB)/triphenyl tetrazolium chloride (TTC) staining (14). In brief, at the end of reperfusion, $2 \mathrm{ml}$ EB (2\%; Sigma-Aldrich; Merck Millipore) was injected intravenously following ligation of the LAD. The heart was removed immediately and stored at $-20^{\circ} \mathrm{C}$ for $20 \mathrm{~min}$. The tissues were sectioned into five transverse slices perpendicular to the long axis ( $2 \mathrm{~mm}$ thick). The slices were incubated in a $1 \%$ TTC solution at $37^{\circ} \mathrm{C}$ for $20 \mathrm{~min}$. The EB-stained area represented the area with normal blood supply. The infarct area (IA) was paler and the area stained red represented the viable tissue at risk. The IA and area at risk were analyzed using Image J software (version 1.44; National Institutes of Health, Bethesda, MD, USA).

Measurements of cardiac troponin $T(c T n-T)$ and creatinine kinase-MB $(C K-M B)$. Blood samples were collected from the rats at the end of reperfusion and were centrifuged at $3,000 \mathrm{xg}$ for $10 \mathrm{~min}$ at $4^{\circ} \mathrm{C}$. The activities of cTn-T and CK-MB in the plasma were estimated using commercially available assay kits (Shanghai XinFan Biotechnology Co., Ltd., Shanghai, China).

Morphological changes in the myocardium. The hearts were collected at the end of reperfusion. Following excision of the superfluous tissue, the left ventricular tissue was fixed in $4 \%$ paraformaldehyde at room temperature for $24 \mathrm{~h}$. Subsequently, the fixed tissues were embedded in paraffin and sectioned at $4 \mu \mathrm{m}$ thicknesses. The myocardial sections were stained with hematoxylin and eosin at room temperature for $2 \mathrm{~min}$ and visualized under a light microscope (magnification, x200; Nikon Corporation, Tokyo, Japan).

Detection of apoptosis. Following dewaxing and rehydration, the myocardial tissue sections ( $4 \mu \mathrm{m}$ thick) were prepared and cell apoptosis was detected using the terminal deoxyribonucleotide transferase mediated dUTP nick end-labeling (TUNEL) assay (Ai Mingkang Bio-Reagent Co., Ltd., Chongqing, China). The stained sections were viewed using fluorescence microscopy (Nikon Corporation) and the TUNEL-positive cells were evaluated at $\mathrm{x} 400$ magnification.

Immunohistochemistry staining. Following dewaxing and rehydraton, the myocardial tissue sections ( $4 \mu \mathrm{m}$ thick) were blocked with 5\% bovine serum albumin (BSA; Sigma-Aldrich; Merck Millipore) and incubated with anti-Bax antibody (1:100) or anti-Bcl-2 antibody (1:100) overnight at $4^{\circ} \mathrm{C}$. Subsequently the sections were incubated with the HRP-conjugated secondary antibody (1:200) at room temperature for $30 \mathrm{~min}$ and visualized under a light microscope (magnification, x200; Nikon Corporation).

Western blot analysis. Proteins were extracted from the myocardial tissues using lysis buffer containing protease (Beyotime Institute of Biotechnology, Haimen, China) and phosphatase inhibitors (Cell Signaling Technology, Inc., Danvers, MA, USA) under homogenization, and the concentrations of protein were measured using a Bicinchoninic Acid Protein Assay kit (Beyotime Institute of Biotechnology). Following separation by $12 \%$ SDS-PAGE, a total of $20 \mu \mathrm{g}$ proteins were transferred onto a polyvinylidene fluoride membrane, blocked with $5 \%$ BSA at room temperature for $1 \mathrm{~h}$, and incubated with specific primary antibodies $(1: 1,000)$ overnight at $4^{\circ} \mathrm{C}$. Subsequently, immunoreactive bands were incubated with a HRP-conjugated secondary antibody $(1: 1,000)$ at room temperature for $1 \mathrm{~h}$ and detected using enhanced chemiluminescence (EMD Millipore, Billerica, MA, USA).

Statistical analysis. Data are expressed as the mean \pm standard deviation. GraphPad Prism software (version 5.0; 


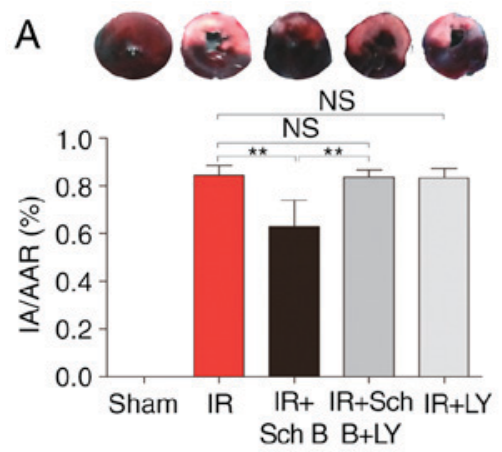

B

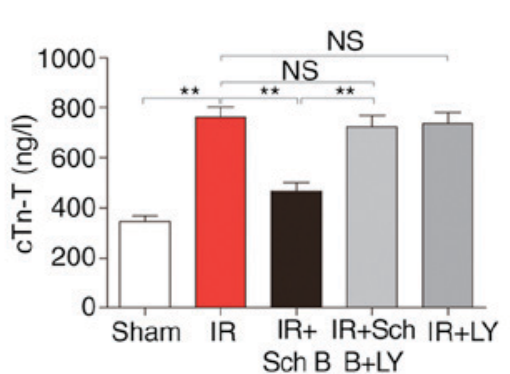

C

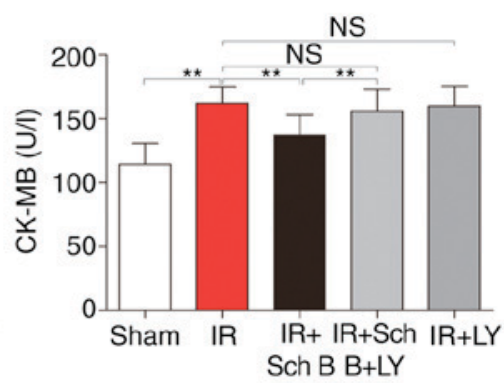

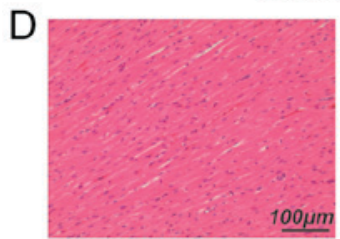

Sham

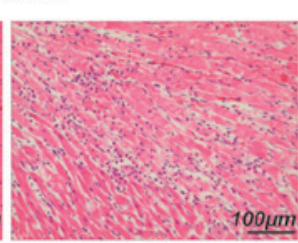

IR

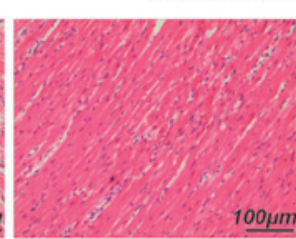

IR+Sch B

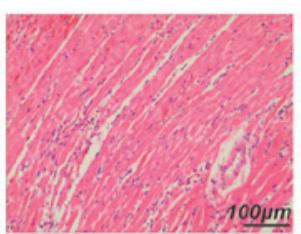

$\mathrm{IR}+\mathrm{Sch} \mathrm{B}+\mathrm{LY}$

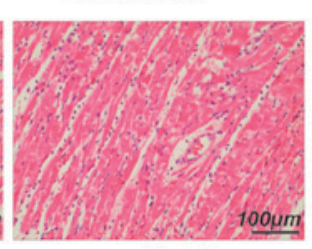

$\mathrm{IR}+\mathrm{LY}$

Figure 1. Effects of Sch B and LY on the area of myocardial infarction ( $\mathrm{n}=6$ for each group), serum-specific cardiac injury biomarkers ( $\mathrm{n}=8$ for each group) and myocardial tissue. (A) Representative images of tetrazolium chloride and Evans Blue staining of sections of the heart. Blue, not at risk; paler, IA; paler and red, AAR. The bar graph represents the percentage of IA vs. AAR. AAR, as a percentage of the area of the whole left ventricle did not differ among the groups. (B) Serum levels of cTn-T. (C) Serum levels of CK-MB. (D) Hematoxylin and eosin staining of myocardial tissue. Data are presented as the mean \pm standard deviation. ${ }^{* *} \mathrm{P}<0.05$. Sch B, schisandrin B; LY, LY294002; IR, ischemia/reperfusion; IA, infarct area; AAR, area at risk; cTn-T cardiac troponin T; CK-MB, creatinine kinase-MB; NS, not significant.

A

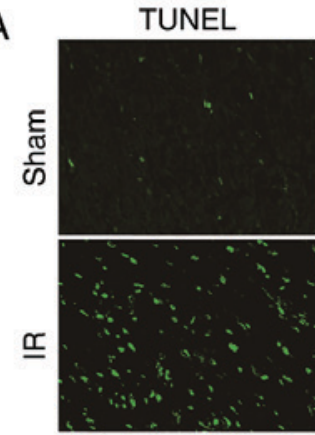

๓
엄
$\stackrel{+}{ \pm}$
$\cong$

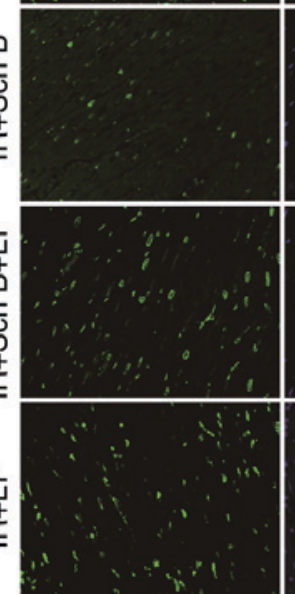

DAPI
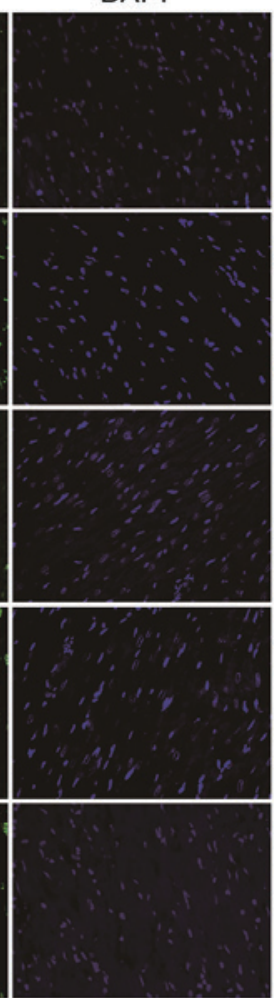

Merge
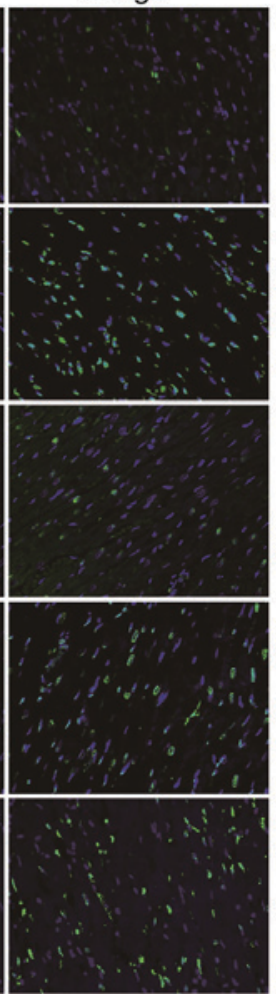

B

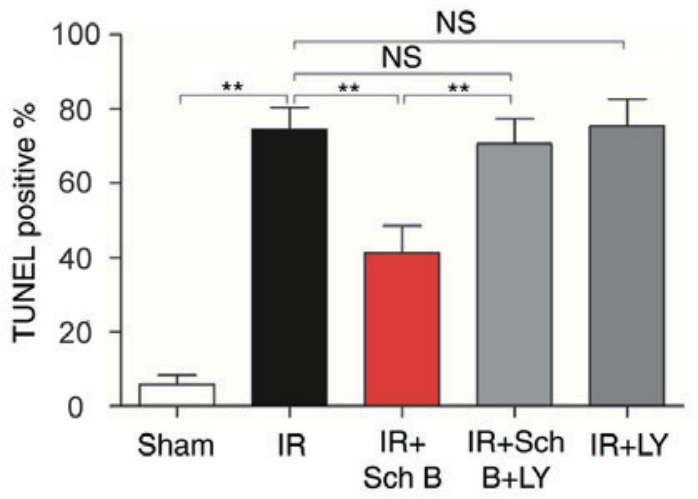

Figure 2. Effects of Sch B and LY on myocardial apoptosis ( $\mathrm{n}=4$ for each group). (A) Representative images (magnification, $\mathrm{x} 400$ ) of TUNEL staining in different groups. Total nuclei were labeled with DAPI (blue), and apoptotic nuclei were detected by TUNEL staining (green). (B) Quantitative analysis of TUNEL-positive cells. Data are presented as the mean \pm standard deviation. ${ }^{* *} \mathrm{P}<0.05$. Sch B, schisandrin B; LY, LY294002; TUNEL, terminal deoxyribonucleotide transferase mediated dUTP nick end-labeling; NS, not significant.

GraphPad Software, Inc., La Jolla, CA, USA) was used for statistical analysis. One-way analysis of variance and Student-Newman-Keuls test were used. $\mathrm{P}<0.05$ was considered to indicate a statistically significant difference.

\section{Results}

Myocardial infarction size. Representative images of the TTC and EB staining for the rats in each group are shown in Fig. 1. 
A

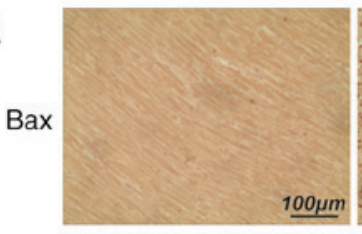

Sham

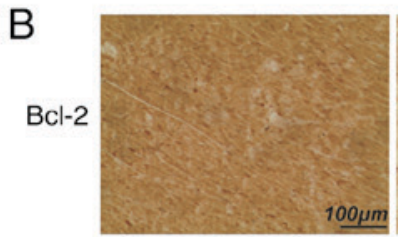

Sham

C

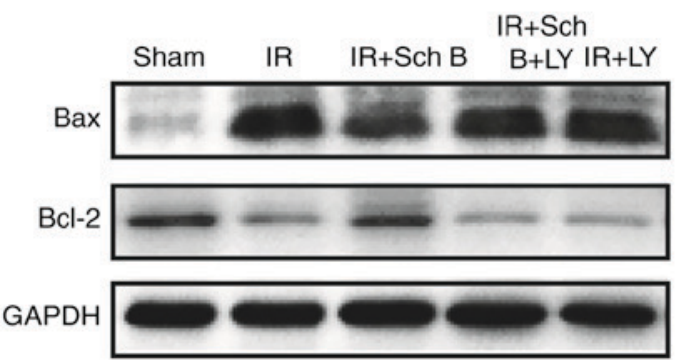

$\mathrm{E}$

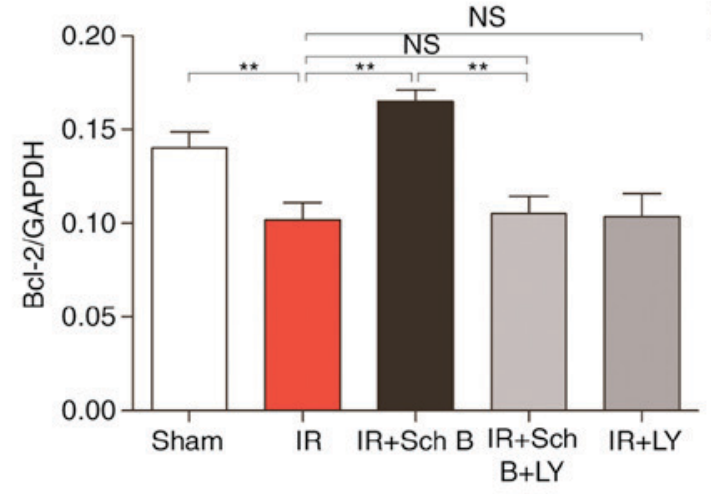

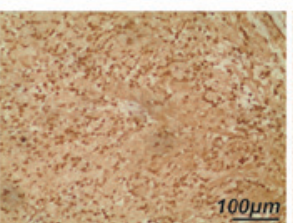

IR

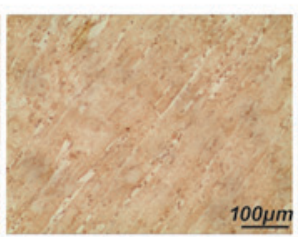

IR

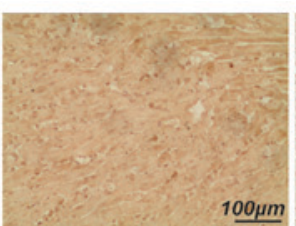

IR+Sch B

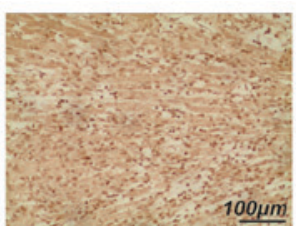

IR+Sch B+LY

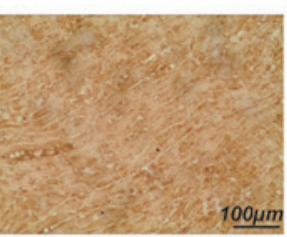

IR+Sch B

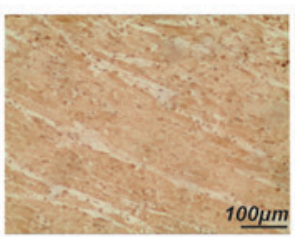

$\mathrm{IR}+\mathrm{Sch} \mathrm{B}+\mathrm{LY}$

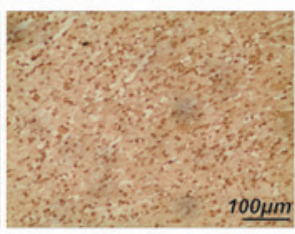

IR+LY

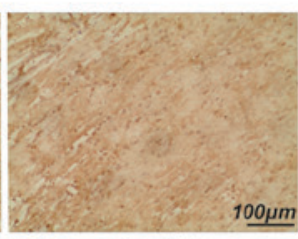

IR+LY

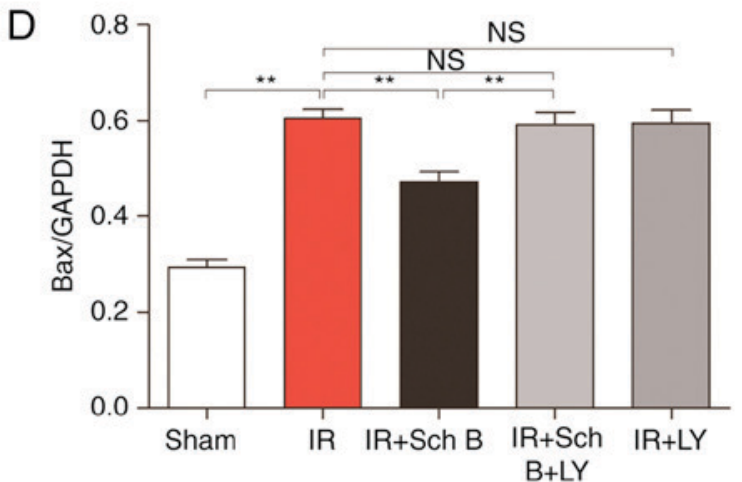

$\mathrm{F}$

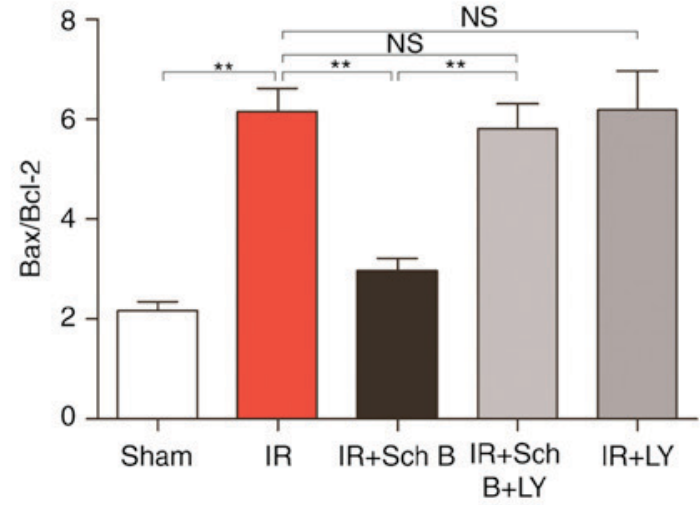

Figure 3. Effects of Sch B and LY on the protein expression of Bax and Bcl-2 in the myocardium. (A) Immunohistochemical analysis of Bax (magnification, x200). (B) Immunohistochemical analysis of Bcl-2 (magnification, x200). (C) Immunoblot of myocardial protein expression levels of Bax and Bcl-2. (D) Densitometry analysis of the protein expression of Bax. (E) Densitometry analysis of the protein expression of Bcl-2. (F) Densitometry analysis of the ratio of Bax/Bcl-2. Data are presented as the mean \pm standard deviation. ${ }^{* *} \mathrm{P}<0.05$. Sch B, schisandrin B; LY, LY294002; IR, ischemia/reperfusion; Bcl-2, B-cell lymphoma 2; Bax, Bcl-2-like protein 4; NS, not significant.

Compared with the control rats, Sch B administration led to a significant reduction in myocardial infarction size $(62.8 \pm 11.2$ vs. $84.5 \pm 4.1 \%$; $\mathrm{P}<0.05)$. However, the protective effect of the medication was eliminated by LY294002 treatment (I/R+Sch B+LY group, $83.8 \pm 2.9 \%$ ), compared with the I/R+ Sch B group $(62.8 \pm 11.2 \%$; $\mathrm{P}<0.05$; Fig. $1 \mathrm{~A})$.

Plasma $c T n-T$ and $C K-M B$. Compared with the sham-operation rats, the levels of myocardial infarction-associated markers in plasma samples were distinctly increased in the control rats. Consistently, Sch B treatment reduced the levels of cTn-T, compared with that in the control rats $(468.3 \pm 92.0$ vs. $761.8 \pm 114.0 \mathrm{ng} / \mathrm{l}$, respectively; $\mathrm{P}<0.05$; Fig. $1 \mathrm{~A})$ and reduced the level of CK-MB, compared with that in the control rats $(136.8 \pm 16.3$ vs. $162.1 \pm 12.9 \mathrm{U} / 1$, respectively;
$\mathrm{P}<0.05$ Fig. 1B). However, the inhibitory effect of Sch $\mathrm{B}$ on the level of cTn-T was eliminated by LY294002 administration, compared with that in the Sch B group (468.3 \pm 92.0 vs. $725.6 \pm 122.2 \mathrm{ng} / 1$, respectively; $\mathrm{P}<0.05)$, with the same observed for CK-MB (136.8 \pm 16.3 vs. $155.7 \pm 17.2 \mathrm{U} / 1$, respectively; $\mathrm{P}<0.05$; Fig. 1).

Morphological changes in the myocardium. Compared with the hearts of the sham group, disorganization of cell structures was observed in the myocardium of untreated rats. Cardiomyocyte necrosis and inflammatory cell infiltration were decreased in the Sch B treatment group, compared with the untreated rats. However, treatment with LY294002 eliminated the protective effects of Sch B in myocardial injury (Fig. 1D). 

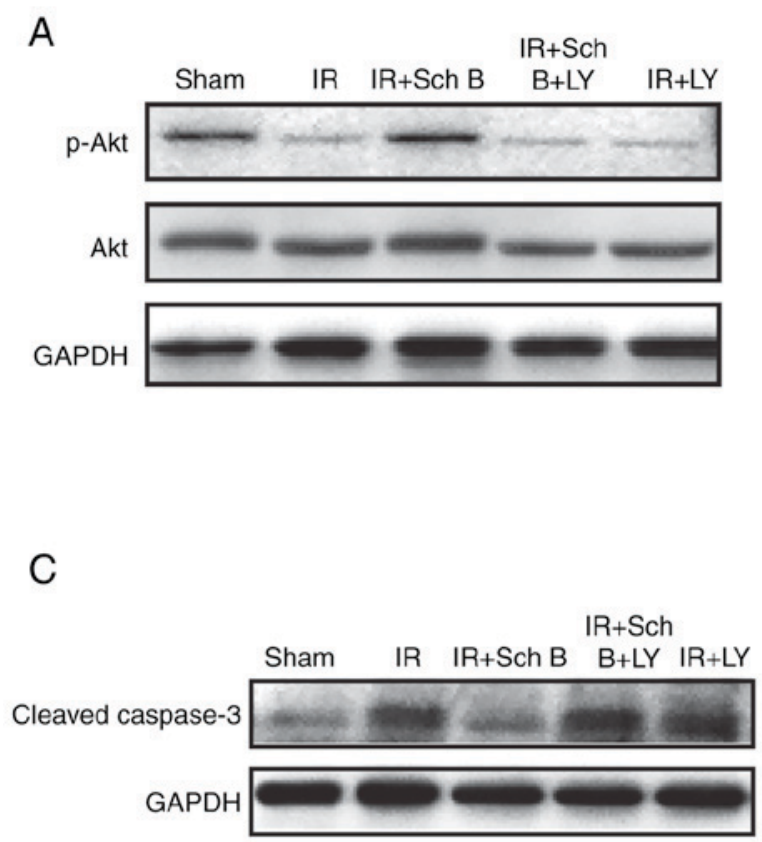
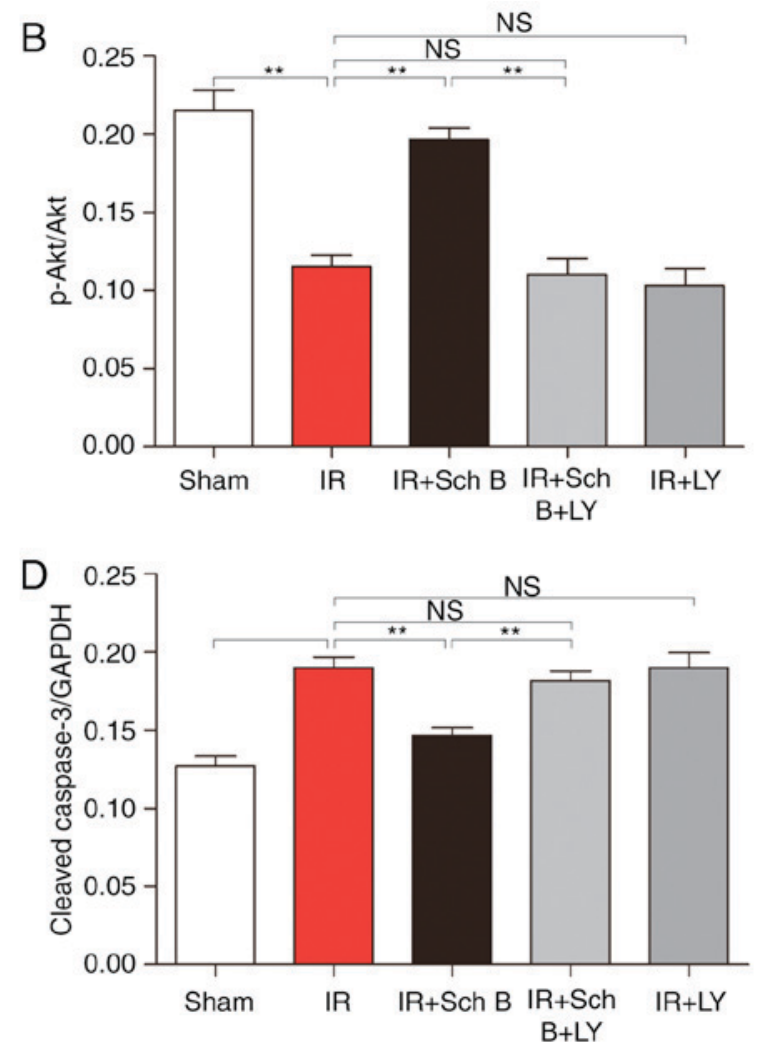

Figure 4. Effects of Sch B and LY on the protein expression of p-Akt and cleaved caspase-3 in the myocardium (n=6 for each group). (A) Immunoblot of the myocardial protein expression of p-Akt. (B) Densitometry analysis of the protein expression of p-Akt. (C) Immunoblot of the myocardial protein expression of cleaved caspase-3. (D) Densitometry analysis of the protein expression levels of cleaved caspase-3. Data are presented as the mean \pm standard deviation. ${ }^{* * *} \mathrm{P}<0.05$. Sch B, schisandrin B; LY, LY294002; IR, ischemia/reperfusion; p-Akt, phosphorylated Akt; NS, not-significant.

Myocardial apoptosis. The results of the TUNEL assay revealed that the number of TUNEL-positive cells was higher in the untreated rats $(74.50 \pm 5.97 \%)$, compared with that in the sham group $(5.75 \pm 2.50 \%)$. Treatment with Sch B reduced the number of TUNEL-positive nuclei stained $(41.25 \pm 7.27 \%$; $\mathrm{P}<0.05)$. However, this anti-apoptotic effect of Sch B was attenuated by LY294002 administration (41.25 \pm 7.27 vs. $70.50 \pm 6.76 \%$, respectively; $\mathrm{P}<0.05)$, as shown in Fig. $2 \mathrm{~A}$ and $\mathrm{B}$.

Immunohistochemical staining. Compared with the untreated rats, the concentrations of Bax were significantly decreased and the concentrations of Bcl-2 were increased in the Sch B treatment groups, however, these effects of Sch B were attenuated by LY294002 treatment (Fig. 3A-F).

Western blot analysis. Treatment with Sch B significantly increased the concentrations of $\mathrm{p}$-Akt, compared with that in the untreated rats $(0.20 \pm 0.02$ vs. $0.12 \pm 0.02$; $\mathrm{P}<0.05$; Fig. $4 \mathrm{~A}$ and B). However, the administration of LY294002, an inhibitor of PI3K, attenuated the Sch B-induced upregulation of myocardial p-Akt in the $\mathrm{I} / \mathrm{R}+\mathrm{Sch} \mathrm{B}+\mathrm{LY}$ group $(0.11 \pm 0.03)$. The concentrations of cleaved caspase- 3 in the myocardium of the $\mathrm{Sch} B+\mathrm{I} / \mathrm{R}$ group were also lower, compared with those in the I/R and Sch B+LY+I/R groups (Fig. 4C and D).

\section{Discussion}

The results of the present study revealed that treatment with the natural medicinal monomer, Sch B, reduced the infract size induced by $\mathrm{I} / \mathrm{R}$. The increased levels of cTn-T and CK-MB were also attenuated by treatment with Sch B, and treatment with $\mathrm{Sch} B$ reduced the number of TUNEL-positive nuclei stained. These results indicated that Sch B exerted protective effects in the myocardium via the decrease in TUNEL-positive cells, which indicated that the cardioprotective effects of Sch B were associated with cardiomyocyte apoptosis.

Cardiomyocyte apoptosis is important in the progress of myocardial infarction and cardiac dysfunction following ischemia (15). As an important regulator, the $\mathrm{Bcl}-2$ protein family is considered to be involved in the myocardial cell apoptotic pathway (16). In addition, Bcl-2 has been shown to have several effects, including the prevention of mitochondria from releasing cytochrome $c$ and inhibiting caspase activity, whereas Bax is known to increase cell apoptosis $(16,17)$. Consequently, the Bax/Bcl-2 ratio affects apoptotic signaling. In the present study, it was confirmed that Sch B decreased the $\mathrm{Bax} / \mathrm{Bcl}-2$ ratio, which suggested that the anti-apoptotic effect of Sch B was associated with the Bcl-2 protein family.

Additionally, the caspase family is considered to be involved in the cell apoptotic process, with caspase- 3 being an essential protease in the apoptotic cascade reaction (18). When caspase-3 is activated, it becomes cleaved caspase-3. In the present study, the results showed that the concentrations of cleaved caspase-3 in the Sch B-pretreated rats were lower, compared with those in the untreated rats, which indicated that the anti-apoptotic effect of Sch B was associated with the caspase family. 
The activation of the PI3K/Akt-dependent pathway is also considered to be involved in myocardial tissue protection and the inhibition of I/R-induced cardiomyocyte apoptosis (19-21) via involvement of the caspase family and the incremental expression of Bcl-2 (22). In the present study, the results showed that the concentrations of p-Akt in the Sch B-pretreated rats were higher, compared with those in the untreated rats. Of note, the use of LY294002 in the present study revealed that the inhibitory action of PI3K eliminated the protective effects of Sch B. Taken together, these results suggested that the potential benefits of Sch B in I/R injury were likely mediated by the activation of PI3K/Akt pathways via involvement of the caspase family and the Bcl-2 protein family.

It has been reported that $\mathrm{Sch} B$ administration in an isolated perfused heart preparation protected the myocardium from $\mathrm{I} / \mathrm{R}$ injury by decreasing mitochondrial sensitivity to the permeability transition induced by calcium ions (10). Additionally, it has been shown that the activation of Akt is vital in maintaining mitochondrial morphology and function, and modulating the sensitivity of mitochondrial permeability transition pore opening (23). Therefore, it is likely that Sch B has a protective effect on myocardial tissues via the PI3K/Akt pathway.

The limitations of the present study require consideration when interpreting the results. First, the investigation is likely to be improved by the addition of a control group treated with DMSO only. Secondly, as the use of TUNEL staining for the detection of apoptosis is controversial, further investigations are required to validate the data. Thirdly, the effects of Sch B on cardiac function require evaluation in further investigations, and other signaling pathways involved in the myocardial protective effects of Sch B require identification.

In conclusion, the present study revealed that Sch B exerted cardioprotective effects by reducing cardiomyocyte apoptosis in an in vivo model. These cardioprotective effects of Sch B required activation of the PI3K/Akt signaling pathway.

\section{Acknowledgements}

The present study was funded by the Science and Technology Bureau of Lishui (grant no. 2012cxtd10).

\section{References}

1. Lapointe-Shaw L and Bell CM: Acute myocardial infarction. $\mathrm{Br}$ Med J 348: f7696, 2014.

2. Fröhlich GM, Meier P, White SK, Yellon DM and Hausenloy DJ: Myocardial reperfusion injury: Looking beyond primary PCI. Eur Heart J 34: 1714-1722, 2013.

3. Iliodromitis K, Farmakis D, Andreadou I, Zoga A, Bibli SI, Manolaki T, Dagres N, Iliodromitis EK, Anastasiou-Nana M and Kremastinos DT: Various models of cardiac conditioning in single or sequential periods of ischemia: Comparative effects on infarct size and intracellular signaling. Int J Cardiol 168 1336-1341, 2013.
4. Lam PY and Ko KM: Schisandrin B as a hormetic agent for preventing age-related neurodegenerative diseases. Oxid Med Cell Longev 2012: 250825, 2012.

5. Panossian A and Wikman G: Pharmacology of Schisandra chinensis Bail: An overview of Russian research and uses in medicine. J Ethnopharmacol 118: 183-212, 2008.

6. Chiu PY, Leung HY, Poon MK, Lee SS and Ko KM: Schisandrin $\mathrm{B}$ induced antioxidant response is partly mediated by cytochrome P-4502E1 catalyzed reaction in mouse liver. Mol Cell Biochem 293: 87-92, 2006.

7. Chang CY, Chen YL, Yang SC, Huang GC, Tsi D, Huang CC, Chen JR and Li JS: Effect of schisandrin B and sesamin mixture on $\mathrm{CCl}(4)$-induced hepatic oxidative stress in rats. Phytother Res 23: 251-256, 2009.

8. Lee TH, Jung CH and Lee DH: Neuroprotective effects of Schisandrin B against transient focal cerebral ischemia in Sprague-Dawley rats. Food Chem Toxicol 50: 4239-4245, 2012.

9. Chen N, Chiu PY and Ko KM: Schisandrin B enhances cerebral mitochondrial antioxidant status and structural integrity, and protects against cerebral ischemia/reperfusion injury in rats. Biol Pharm Bull 31: 1387-1391, 2008.

10. Chiu PY, Leung HY, Siu AH, Poon MK and Ko KM: Schisandrin $\mathrm{B}$ decreases the sensitivity of mitochondria to calcium ion induced permeability transition and protects against ischemia/reperfusion injury in rat hearts. Acta Pharmacol Sin 28: 1559-1565, 2007.

11. Qiu Y, Li P and Ji C: Cell death conversion under hypoxic condition in tumor development and therapy. Int J Mol Sci 16: 25536-25551, 2015.

12. Zhao GX, Pan H, Ouyang DY and He XH: The critical molecular interconnections in regulating apoptosis and autophagy. Ann Med 47: 305-315, 2015.

13. Hu W, Zhang P, Gu J, Yu Q and Zhang D: NEDD4-1 protects against ischaemia/reperfusion-induced cardiomyocyte apoptosis via the PI3K/Akt pathway. Apoptosis 22: 437-448, 2017.

14. Black SC and Rodger IW: Methods for studying experimental myocardial ischemic and reperfusion injury. J Pharmacol Toxicol Methods 35: 179-190, 1996.

15. Gottlieb RA and Engler RL: Apoptosis in myocardial ischemia-reperfusion. Ann New York Acad Sci 874: 412-426, 1999.

16. Kumar D and Jugdutt BI: Apoptosis and oxidants in the heart. J Lab Clin Med 142: 288-297, 2003.

17. Othman AI, Elkomy MM, El-Missiry MA and Dardor M: Epigallocatechin-3-gallate prevents cardiac apoptosis by modulating the intrinsic apoptotic pathway in isoproterenol-induced myocardial infarction. Eur J Pharmacol 794: 27-36, 2017.

18. Zhou PY, Zhang Z, Guo YL, Xiao ZZ, Zhu P, Mai MJ and Zheng SY: Protective effect of antiapoptosis potency of prolonged preservation by desiccation using high-pressure carbon monoxide on isolated rabbit hearts. Transplant Proc 47: 2746-2751, 2015.

19. Lu Y,Zhou J, Xu C, Lin H, Xiao J, Wang Z and Yang B: JAK/STAT and PI3K/AKT pathways form a mutual transactivation loop and afford resistance to oxidative stress-induced apoptosis in cardiomyocytes. Cell Physiol Biochem 21: 305-314, 2008.

20. Bharti S, Golechha M, Kumari S, Siddiqui KM and Arya DS: Akt/GSK-3 $\beta /$ NOS phosphorylation arbitrates safranal-induced myocardial protection against ischemia-reperfusion injury in rats. Eur J Nutr 51: 719-727, 2012.

21. Bharti S, Singh R, Chauhan SS, Hussain T, Al-Attas OS and Arya DS: Phosphorylation of Akt/GSK-3//eNOS amplifies 5-HT2B receptor blockade mediated anti-hypertrophic effect in rats. FEBS Lett 586: 180-185, 2012.

22. Ge N, Liu C, Li G, Xie L, Zhang Q, Li L, Hao N and Zhang J: Hydrosulfide attenuates acute myocardial ischemic injury through the glycogen synthase kinase- $3 \beta / \beta$-catenin signaling pathway. Int J Mol Med 37: 1281-1289, 2016.

23. Ong SB, Hall AR, Dongworth RK, Kalkhoran S, Pyakurel A, Scorrano L and Hausenloy DJ: Akt protects the heart against ischaemia-reperfusion injury by modulating mitochondrial morphology. Thromb Haemost 113: 513-521, 2015. 